\title{
Wheat Resistance to Fusarium Head Blight is Associated With Changes in Photosynthetic Parameters
}

\begin{abstract}
Shimin Yang, State Key Laboratory for Biology of Plant Diseases and Insect Pests, Institute of Plant Protection, Chinese Academy of Agricultural Sciences (CAAS), Beijing 100193, China; and Provincial Key Laboratory of Plant Breeding and Genetics, Sichuan Agricultural University, Chengdu, Sichuan 611130, China; Xin Li, Provincial Key Laboratory of Plant Breeding and Genetics, Sichuan Agricultural University, Chengdu, Sichuan 611130, China; Wanquan Chen and Taiguo Liu, State Key Laboratory for Biology of Plant Diseases and Insect Pests, Institute of Plant Protection, Chinese Academy of Agricultural Sciences (CAAS), Beijing 100193, China; Shengfu Zhong, Lixia Ma, Min Zhang, Huaiyu Zhang, and Dailong Yu, Provincial Key Laboratory of Plant Breeding and Genetics, Sichuan Agricultural University, Chengdu, Sichuan 611130, China; and Peigao Luo, State Key Laboratory for Biology of Plant Diseases and Insect Pests, Institute of Plant Protection, Chinese Academy of Agricultural Sciences (CAAS), Beijing 100193, China; and Provincial Key Laboratory of Plant Breeding and Genetics, Sichuan Agricultural University, Chengdu, Sichuan 611130, China
\end{abstract}

\begin{abstract}
Yang, S. M., Li, X., Chen, W. Q., Liu, T. G., Zhong, S. F., Ma, L. X., Zhang, M., Zhang, H. Y., Yu, D. L., and Luo, P. G. 2016. Wheat resistance to Fusarium head blight is associated with changes in photosynthetic parameters. Plant Dis. 100:847-852.

Fusarium head blight (FHB) is an important wheat disease worldwide; however, its effects on the physiological parameters in plants with different levels of FHB resistance remain unclear. Here, we evaluated the effects of Fusarium graminearum infection on yield and the photosynthesisassociated parameters $P_{n}, G_{s}$, and $C_{i}$ of wheat flag leaves and determined the influence of FHB resistance. The FHB-resistant wheat genotype L699 and its susceptible sister line L661 were point- and spray-inoculated. Photosynthesis-associated parameters were subsequently measured using a modulated photosynthesis system, and FHB intensity was evaluated. Compared with L661, FHB caused more significant reductions in the net

photosynthetic rate and stomatal conductance of flag leaves in L699. However, FHB caused a larger reduction in the 1000-grain weight and total grain weight per spike in L661 compared with L699. Independence sample $t$ test showed that FHB resistance was significantly higher in L699 compared with L661. We concluded that under the conditions of the present study, FHB had a significantly greater effect on net photosynthesis in the resistant line compared with the susceptible line; however, it had a greater impact on yield components in the susceptible line. These results provide a new insight into the physiological cost of host resistance to FHB.
\end{abstract}

Wheat (Triticum aestivum L.) accounts for approximately $20 \%$ of the calories consumed by humans. Fusarium head blight (FHB), mainly caused by Fusarium graminearum Schwabe and $F$. culmorum WG Smith, is an economically important fungal disease in many wheat-growing areas and seriously threatens wheat production worldwide (21). Several FHB outbreaks in wheat and barley (Horderum vulgare L.) from 1991 to 1997 resulted in estimated total direct losses of approximately $\$ 1.3$ billion and cumulative losses of $\$ 4.8$ billion in the United States (1). From 1998 to 2000, the direct economic losses due to FHB infection of wheat in the northern Great Plains and the central United States were estimated at $\$ 734$ million (24), while the total economic losses due to FHB reached $\$ 2.7$ billion in the United States during the same time period (23).

In China, FHB epidemics have historically occurred in the middle to lower valleys of the Yangtze River, including Jiangsu, Zhejiang, Anhui, and Hubei provinces, and the northeast spring wheatgrowing area. In these areas, seven severe and 10 moderate epidemics of FHB were reported from 1951 to 1990 (35). In recent years, FHB has become increasingly severe in the southwest region of China (36). For example, in 2004, the epidemic area reached 292,000 ha in Sichuan, and the proportion of FHB-infected spikes ranged from 10 to $61 \%$ (29). A serious FHB epidemic occurred in 2012 in the Yellow and Huai River valleys, including southern Shandong, Henan, Shanxi, Hebei, and northern parts of Jiangsu,

Corresponding author: Peigao Luo, Email: lpglab@sicau.edu.cn

S. M. Yang and X. Li contributed equally to this work.

Accepted for publication 4 December 2014

http://dx.doi.org/10.1094/PDIS-04-14-0398-RE

(C) 2016 The American Phytopathological Society
Anhui, and Hubei provinces, which are the most important wheatgrowing areas of China (14). Another complication of FHB is the accumulation of mycotoxins produced by Fusarium spp., especially deoxynivalenol, in wheat grain, which can cause acute food poisoning in humans and animals after they eat infected grain $(7,38)$.

Although crop management practices and application of chemicals may reduce the damage caused by FHB, the development of genetically resistant cultivars is the most effective approach to combat this disease (1). In the past, efforts have been directed toward the identification of new sources of resistant germplasm because such material is required for the development of FHB-resistant cultivars $(4,22)$. However, few studies have been conducted to investigate the physiological response of wheat to Fusarium inoculation and the influence of FHB resistance on this response.

The infection of plant tissue with fungal pathogens is closely linked to changes in metabolic pathways such as photosynthesis (9). Different plant organs have diverse tasks and biological functions. For example, one of the crucial functions of the leaf as a "source" tissue is to synthesize energy-rich molecules via photosynthesis for the transport of carbon, whereas a developing seed is a carbohydrate "sink" (30). It is well known that the FHB pathogen infects spike tissues and thus affects kernel development (5). Kernels are the most important nonphotosynthetic sink of the wheat plant. The relationship between photosynthetic sources and nonphotosynthetic sinks is maintained in a dynamic balance $(15,19)$, and changes in this balance that occur when plant pathogens attack are likely to manifest as changes in photosynthesis-related parameters due to the regulation of cellular signaling homeostasis $(27,34)$. In tomato plants infected with $F$. oxysporum, the level of chlorophyll fluorescence has been used to indicate disease severity (32). This suggests that certain physiological phenotypes could be associated with disease resistance, and measurements of such phenotypes could presumably provide some important information about the effects of host FHB resistance on the physiological mechanisms of the wheat plant. In addition, functional and 
comparative bioinformatics studies have shown that many genes involved in photosynthesis play key roles in protection against both $F$. graminearum and $F$. culmorum $(9,11,12)$. These findings indicate that wheat FHB resistance is related to photosynthetic parameters. However, to date, the relationship between FHB and photosynthetic parameters has not been determined. Understanding this relationship will be valuable for determining the costs of FHB resistance.

In the present study, a new FHB-resistant wheat line L699 and its susceptible sister line L661 were used to study the effects of $F$. graminearum infection on photosynthesis-associated parameters and yield components. The use of sister lines contrasting in their reactions to FHB for studying the effect of FHB resistance on these parameters significantly reduces the differences in genetic backgrounds between the two lines. Photosynthetic traits, including the net photosynthetic rate $\left(P_{n}\right)$, stomatal conductance $\left(G_{s}\right)$, and intercellular $\mathrm{CO}_{2}$ concentration $\left(C_{i}\right)$, were investigated using the $\mathrm{Li}-6400$ photometer system. In addition, grain weight per spike and 1000-kernel weight were also measured because they may also be affected by FHB (28). The overall aims of the present study were to i) determine the changes in photosynthetic parameters and yield components after inoculation with $F$. graminearum, ii) investigate the physiological effects of $F$. graminearum inoculation on wheat flag leaves, and iii) determine whether changes in physiological responses to FHB were influenced by FHB resistance.

\section{Materials and Methods}

Plant material and resistance screening. Two wheat sister lines, L661 and L699 (Reg. No. GP-974, PI 672540), were selected from a segregating $\mathrm{F}_{6: 7}$ recombinant inbred line of the cross MY11/YU25 (13). L699 has strong resistance to both stripe rust caused by Puccinia striiformis Westend. f. sp. tritici and powdery mildew caused by Erysiphe graminis f. sp. tritici, which is conferred by the $Y r Y U 25$ (YrL693) and Pm40 genes, respectively, from YU25 $(10,16,18)$. L661 is resistant only to powdery mildew. In addition, field observations showed that L699 is highly resistant to FHB and that L661 is FHB susceptible (36).

More than 600 seeds of each genotype were sown during the 2009-2010 and 2010-2011 wheat-growing seasons in a field at the Ya'an Agricultural Research Station $\left(27^{\circ} 17^{\prime} \mathrm{N}\right.$ latitude, $120^{\circ} 16^{\prime} \mathrm{E}$ longitude, approximately 620 meters above sea level) of Sichuan Agricultural University in southwest China, where the climate is temperate and rainy with a yearly average temperature of 15 to $17^{\circ} \mathrm{C}$ and yearly average precipitation of $1,520 \mathrm{~mm}$ (19). All experiments used a randomized complete block design with three replications. Each plot consisted of 3-m-long rows with $0.5-\mathrm{m}$ spacing between rows, and $30 \mathrm{~cm}$ between plants.

In this study, we sprayed a macroconidial suspension containing 200 macroconidia/ $\mu 1$ on the spikes of approximately 200 plants. These plants were randomly chosen from each plot at anthesis to screen for type I resistance. A single spore-derived isolate of $F$. graminearum No. 4 (kindly provided by Professor Zhengqiang Ma at Nanjing Agricultural University, Nanjing, Jiangsu Province, China) was used to prepare conidial suspensions as follows: the F. graminearum conidia were placed into $\mathrm{V} 8$ juice agar and grown for 1 to 2 weeks at room temperature. Next, falcate conidia collected from the plates were transferred into liquid mung bean medium and cultured at $25^{\circ} \mathrm{C}$ for 3 days with shaking at $150 \mathrm{rpm}$. Finally, the conidial suspension was filtered through Miracloth, centrifuged at 8,000 rpm for $10 \mathrm{~min}$, placed in an ice bucket for transport to the field, and used for inoculation within $5 \mathrm{~h}$. Heads from the main stem were randomly marked to evaluate FHB resistance. Although the number of heads investigated varied depending on the genotype and year, at least 64 spikes were evaluated per genotype in each year. The disease incidence (DI) was calculated as the percentage of heads with visible symptoms for two independent experiments (in 2010 and 2011 cropping seasons). Diseased spikelets were counted, and the mean percentage of diseased spikelets (PDS) was calculated at 7, 14, 21, and 28 days after inoculation.

To determine type II resistance, $5 \mu l$ of a macroconidial suspension $(200$ macroconidia/ $\mu \mathrm{l})$ was injected into two opposite florets of a spikelet at early anthesis, and the spikes from the main stem of approximately 200 plants per genotype were randomly marked and used for inoculation. The inoculated spikes were covered with plastic bags for 3 days to maintain high relative humidity. At least 77 spikes per genotype were evaluated in each year. For each genotype, DI and PDS were recorded (including the inoculated spikelets) on a 0 (no disease) to 1 (100\% infected spikelets) scale (with increments of 0.1 ) at $7,14,21$, and 28 days after inoculation. All inoculated spikes were harvested and processed individually by hand to avoid loss of seriously diseased grains. Fusarium-damaged kernels (FDK) were distinguished by their obviously shriveled shape, light weight, and chalky white (or occasionally pink) color. These parameters were also evaluated from 70 randomly selected uninoculated spikes in each plot from each experiment (2010 and 2011 growing seasons).

The area under the disease progress curve (AUDPC) was calculated using the PDS collected from each plant, and the AUDPC value for each genotype was calculated from the mean of all the observed plants using a previously described equation (2).

Measurement of photosynthetic parameters and yield components. Ten plants of each genotype (L661 and L699) were randomly selected from among the spray inoculation, point inoculation, and control plants. Net photosynthetic rates and related physiological parameters of each plant were measured twice at 7 days and 21 days after inoculation, respectively. The mean value of three measurements per plant represented a replicate sample, and the mean from all 10 plants represented the value for a given genotype at a given time point. The measurements were made in the middle of the flag leaf (3). $P_{n}, G_{s}$, and $C_{i}$ were measured using a Li-6400 photometer (LiCor, Lincoln, NE), with air temperatures from 21 to $24^{\circ} \mathrm{C}$ and vapor pressure deficits (VPD) from 0.55 to $0.65 \mathrm{kPa}$. The actinic light intensity was $1,000 \mu \mathrm{mol} \cdot \mathrm{m}^{-2} \cdot \mathrm{s}^{-1}$. Each measurement took approximately $30 \mathrm{~s}$.

The seeds of the plants marked for photosynthetic measurement were harvested individually by hand. The number of seeds per spike and the number of infected seeds per spike were counted, and the percentage of infected seeds was calculated. After the seeds were dried in an oven, grain weight per spike and 1000-grain weight were determined based on the mean of the 10 marked spikes.

Statistical analysis. Significant differences in the mean disease severity, physiological parameters, and yield traits of randomly selected plants from three replications between the two wheat lines (L699 and L611) were determined separately for each inoculation method in each year using an independent samples $t$ test with IBM SPSS Statistics 19 software (SPSS Inc., Chicago, IL). In addition, significant differences in the mean physiological parameters and yield traits between both the point- and spray-inoculated and the uninoculated plants of the same genotypes were also determined by the same program.

\section{Results}

FHB resistance of host plants. All wheat lines showed visible FHB symptoms after either point or spray inoculation. The PDS after 7 and 21 days, DI, AUDPC, and FDK are shown in Table 1. In two independent experiments, $t$ test of the inoculated plants randomly selected from three replications showed that L699 had significantly lower disease severity than L661, and the differences between L699 and L661 for all measurements of FHB infection were significant at $(P<0.01)$. More importantly, some L699 plants did not show obvious symptoms at the inoculation point, whereas all inoculated spikelets of L661 had visible symptoms after 14 days.

Effects of FHB on photosynthetic parameters. Photosynthetic traits are usually evaluated using comprehensive photosynthetic parameters such as $P_{n}, G_{s}$, and $C_{i}$. The data for these parameters are shown in Figure 1. $P_{n}$ is the most important photosynthetic parameter and is most sensitive to stresses such as disease infection. The differences in the photosynthetic parameters between uninoculated L699 and L661 wheat lines were not significant at the $P=0.05$ level in the two independent experiments at both 7 days and 21 days after flowering. Spray-inoculated L699 plants showed a significant $(P<$ $0.01)$ decrease in $P_{n}$ at 7 days in 2010 and at 7 and 21 days in 2011 compared with uninoculated plants, whereas $P_{n}$ was significantly 
Table 1. Disease incidence (DI), percentage of diseased spikelets (PDS), area under the disease progress curve (AUDPC), and Fusarium-damaged kernels (FDK) evaluated by inoculating wheat plants with $F$. graminearum in field experiments in 2010 and $2011^{\mathrm{a}}$

\begin{tabular}{|c|c|c|c|c|c|}
\hline \multirow[b]{2}{*}{ Parameter } & \multirow[b]{2}{*}{ Year } & \multicolumn{2}{|c|}{ Point inoculation } & \multicolumn{2}{|c|}{ Spray inoculation } \\
\hline & & L661 & L699 & L661 & L699 \\
\hline \multirow[t]{2}{*}{$\mathrm{PDS}^{\mathrm{b}}$} & 2010 & $0.096 \pm 0.002$ & 0 & $0.002 \pm 0.001$ & 0 \\
\hline & 2011 & $0.138 \pm 0.004$ & 0 & $0.004 \pm 0.002$ & 0 \\
\hline \multirow[t]{2}{*}{$\mathrm{PDS}^{\mathrm{c}}$} & 2010 & $0.283 \pm 0.013$ & $0.078 \pm 0.005^{* *}$ & $0.075 \pm 0.007$ & $0.048 \pm 0.004 * *$ \\
\hline & 2011 & $0.357 \pm 0.017$ & $0.085 \pm 0.006^{* *}$ & $0.145 \pm 0.012$ & $0.034 \pm 0.007 * *$ \\
\hline \multirow[t]{2}{*}{ AUDPC } & 2010 & $5.829 \pm 0.183$ & $1.248 \pm 0.083^{* *}$ & $2.089 \pm 0.126$ & $1.196 \pm 0.078^{* *}$ \\
\hline & 2011 & $6.731 \pm 0.176$ & $1.487 \pm 0.057 * *$ & $2.394 \pm 0.166$ & $0.974 \pm 0.041 * *$ \\
\hline \multirow[t]{2}{*}{ PDK } & 2010 & $0.097 \pm 0.008$ & $0.062 \pm 0.008 * *$ & $0.043 \pm 0.005$ & $0.020 \pm 0.002 * *$ \\
\hline & 2011 & $0.162 \pm 0.018$ & $0.076 \pm 0.006^{* *}$ & $0.059 \pm 0.007$ & $0.016 \pm 0.003 * *$ \\
\hline \multirow[t]{2}{*}{ DI } & 2010 & 1 & 0.876 & 0.781 & 0.660 \\
\hline & 2011 & 0.983 & 0.934 & 0.834 & 0.592 \\
\hline
\end{tabular}

a In point inoculation experiments, 77 L661 spikes and 105 L699 spikes in 2010, and 89 L661 spikes and 94 L699 spikes in 2011 , were observed. In spray inoculation experiments, 64 L661 spikes and 103 L699 spikes in 2010, and 72 L661 spikes and 81 L699 spikes, were observed. The data are presented as the mean $\pm \mathrm{SE} ; * *=$ significantly different from L661 at $P<0.01$.

b PDS at 7 days after inoculation.

c PDS at 21 days after inoculation.

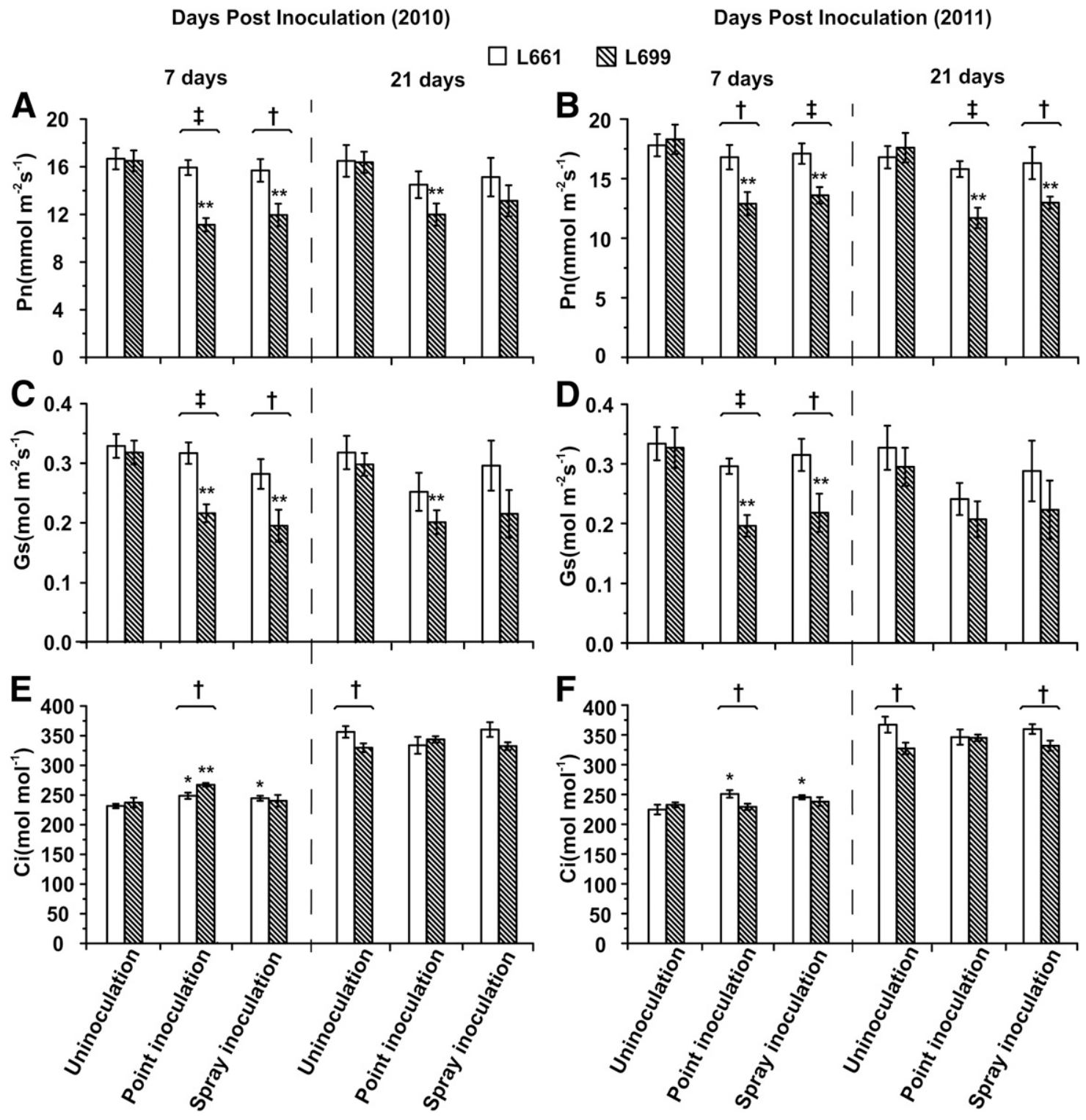

Fig. 1. Fusarium head blight inoculation via both spraying and injection of Fusarium graminearum had different effects on the photosynthetic parameters of wheat plants in two experiments conducted in 2010 and 2011. Net photosynthetic rate $\left(P_{n}\right)$ in $2010(\mathbf{A})$ and $2011(\mathbf{B})$; stomatal conductance $\left(G_{s}\right)$ in $2010(\mathbf{C})$ and $2011(\mathrm{D})$; intercellular $\mathrm{CO}_{2}$ concentration $\left(C_{i}\right)$ in $2010(\mathbf{E})$ and $2011(\mathbf{F})$. The data are presented as the means \pm SE $(n=10) .{ }^{*}=$ significantly different from uninoculated plants of the same genotype at $P<0.05 ;{ }^{* *}=$ significantly different from uninoculated plants of the same genotypes at $P<0.01 ; \dagger=$ significantly different from L661 inoculated with same method at $P<$ $0.05 ;+=$ significantly different from L661 inoculated with same method at $P<0.01$. 
lower $(P<0.01)$ in point-inoculated L699 compared with uninoculated L699 plants at all time points investigated (Fig. 1A and B). Although the change in $P_{n}$ in L661 plants was initially similar to that observed for L699, both spray and point inoculation had little effect on the $P_{n}$ of L661. The reduction in $P_{n}$ observed with spray inoculation in 2010 was not significant $(P=0.461)$. Inoculation had contrasting effects on $P_{n}$ between L661 and L699; the $P_{n}$ of L699 was significantly $(P<0.05)$ lower than that of L661 at 7 days in both 2010 and 2011 and at 21 days in 2011.

$G_{s}$ also plays a key role in plant adaptation to changing environmental conditions and to infection. The $G_{s}$ values were significantly $(P<0.01)$ lower at 7 days after inoculation in both spray- and pointinoculated L699 plants than in uninoculated plants in the two independent experiments, whereas changes in L661 in response to inoculation were not significant at the same time point (Fig. 1C and D). In L699, both spray and point inoculation led to lower $G_{s}$ values compared with the uninoculated L699 plants in both experiments. In L661, inoculation also reduced $G_{s}$; however, the reduction was very small compared with that observed in L699 (Fig. 1C and D). In both experiments, the $G_{s}$ value at 7 days after inoculation was significantly lower in L699 than in L661, regardless of whether inoculation was performed via spraying or injection (Fig. 1C and D). Thus, $G_{s}$ in L699 was more sensitive to infection than $G_{s}$ in L661.

$C_{i}$ is balanced by both $P_{n}$ and $G_{s}$; thus, a comparison of the change in $C_{i}$ with the changes in both $P_{n}$ and $G_{s}$ between genotypes may provide useful information regarding how photosynthetic indices respond to disease stress. $C_{i}$ of the inoculated L661 plants was significantly $(P<0.05)$ higher compared with that of uninoculated plants at 7 days after inoculation; however, the change in inoculated L699 plants was not significant (with the exception of L699 plants inoculated by injection in 2010) (Fig. 1E and F). The effects of $F$. graminearum infection on $C_{i}$ in the two genotypes at 21 days after inoculation were similar, although there were some differences between the two inoculation methods.

Effect of FHB on yield components. Yield components such as 1000 -grain weight and total grain weight per spike are usually useful parameters for determining responses of different genotypes to disease infection. Uninoculated L699 and L661 plants had similar 1000-grain weight and grain weight per spike in the two experiments (Fig. 2). In both experiments, spray inoculation caused a significant
$(P<0.05)$ reduction in 1000-grain weight and grain weight per spike in L661 plants but not in L699 plants (Fig. 2). Although point inoculation led to a significant decrease in 1000-grain weight and grain weight per spike in both L661 and L699, L661 had a greater reduction in these yield components than L699, and L699 had significantly $(P<0.01)$ higher 1000 -grain weight and grain weight per spike than L661 in the point inoculation experiment (Fig. 2). FHB infection by ether spray or point inoculation had a greater negative effect on the yield components in L661 compared with L699.

\section{Discussion}

In the present study, we aimed to characterize the effects of $F$. graminearum infection on photosynthetic and yield traits between two genotypes with contrasting levels of FHB resistance. We also evaluated the physiological cost of host FHB resistance in wheat. Our results show that FHB infection had a smaller effect on photosynthetic parameters and a larger negative effect on yield components in the FHB-susceptible genotype than in the resistant genotype, suggesting that host FHB resistance has a greater physiological cost and a more modest yield cost.

L699 had significantly $(P<0.01)$ lower PDS (at 7 and 21 days after FHB inoculation), DI, AUDPC, and FDK in both experiments than L661, suggesting that L699 had stronger resistance of both type I and type II than L661. Thus, L699 was employed as the resistant genotype and L661 was employed as the susceptible genotype to determine the differential effects of FHB infection on photosynthetic parameters and yield components.

FHB infection affects photosynthetic parameters such as $P_{n}$ and $G_{s}$. Theoretically, the physiological cost of host FHB resistance can be measured by the changes in the photosynthetic parameters after FHB infection. Unfortunately, studies on the physiological cost of FHB resistance are still scarce. It has been shown that $F$. graminearum infection changes the allocation of carbon to various organs (8). Thus, the photosynthetic parameters in flag leaves may be altered when spikes are attacked by $F$. graminearum. Although the location of the $F$. graminearum attack is the spike, this attack would alter the photosynthetic activity via feedback regulation mechanisms. Therefore, differences in the photosynthetic parameters may reveal differences in the costs of FHB resistance in different genotypes after inoculation with $F$. graminearum.
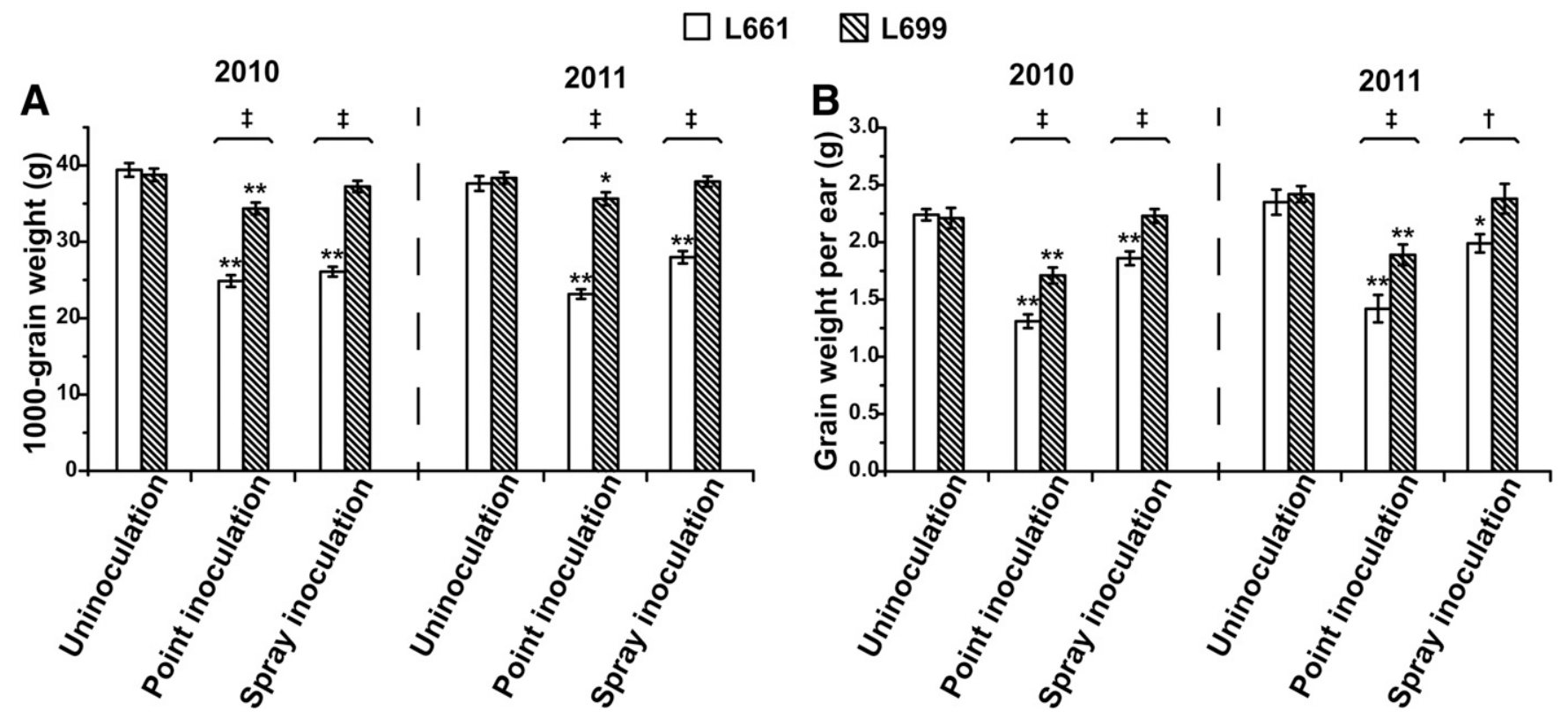

Fig. 2. Inoculation with Fusarium graminearum had a more negative effect on the yield components of susceptible wheat plants (L661) than resistant (L699) plants. (A) 1000-grain weight. (B) Grain weight per spike. The data are presented as the means $\pm \mathrm{SE}$. ${ }^{*}=$ significantly different from uninoculated plants of the same genotype at $P<0.05$; ${ }^{*}=$ significantly different from uninoculated plants of the same genotypes at $P<0.01 ; \dagger=$ significantly different from L661 inoculated with same method at $P<0.05 ;+=$ significantly different from L661 inoculated with same method at $P<0.01$. 
In the two independent experiments (2010 and 2011), $P_{n}$, the most important parameter of photosynthesis, decreased significantly $(P<$ 0.01) in the FHB-resistant line L699 within 7 days after spray and point inoculation, whereas in L661, the reduction in $P_{n}$ was trivial. A previous expression profiling study of the soybean response to infection by Pseudomonas syringae showed similar results, i.e., infection led to a greater reduction in photosynthesis during the resistance response than during the susceptible response (37). This result suggested that host FHB resistance may be associated with a $P_{n}$ cost.

Because stomata play a vital role in the regulation of gas exchange and water loss, stomatal function is likely related to physiological processes such as photosynthesis and respiration as well as the ability of plants to cope with pathogen infection (25). Therefore, $G_{s}$-mediated plant disease resistance has been proposed as the most common form of nonhost resistance associated with plant fitness $(6,26,33) . F$. graminearum infection by either spraying or injection resulted in a significant $(P<0.01)$ decrease in $G_{s}$ in the FHBresistant line L699, but not in the FHB-susceptible line L661, within 7 days of inoculation. $G_{s}$ and the activity of the photosynthetic apparatus are the two main factors influencing $P_{n}$ (19). An analysis of the disease data and the photosynthetic parameters suggests that $P_{n}$ in L661 may be regulated mainly by stomatal factors; however, $P_{n}$ in L699 is not. This actively regulated physiological mechanism has been considered as part of the host resistance mechanism mediated by host resistance genes (33).

In this study, L661 and L699 had similar values for physiological parameters and yield components under the control conditions (without inoculation) due to the similar genetic background of these lines. Indeed, only $39(2.26 \%)$ of the 1,726 loci amplified by 781 SSR primers were polymorphic between the two lines (unpublished data). In L699, the point-inoculated plants showed obvious reductions in $P_{n}$ and $G_{s}$ at 7 and 21 days after inoculation compared with the uninoculated plants, whereas the corresponding changes were very trivial in L661. In addition, the spray-inoculated L699 plants also showed reduced $P_{n}$ and $G_{s}$ at both 7 and 21 days after inoculation compared with the uninoculated plants. Thus, changes in $P_{n}$ and $G_{s}$ between the two genotypes suggested a large physiological cost for host FHB resistance, especially at 7 days after $F$. graminearum infection. At this time point, both $P_{n}$ and $G_{s}$ were significantly $(P<0.05)$ lower in the FHB-resistant line L699 compared with uninoculated L699, whereas the decrease was trivial in L661.

In contrast, 1000-grain weight and grain weight per spike were sharply reduced for both the point- and spray-inoculated plants and the uninoculated control L661 plants, whereas in L699, the reductions were smaller than those in L661. Although F. graminearum infection of FHB-resistant L699 plants resulted in a decrease in photosynthetic parameter values, these plants maintained a higher yield. On the contrary, $F$. graminearum-infected FHB-susceptible L661 plants exhibited higher photosynthetic parameter values, although their yield was lower. This finding may be explained by alterations in the source-sink relationship (31). This result also further indicated that $F$. graminearum infection might activate the nonflag-leaf photosynthetic organs, such as the spike (19), which may serve as a "buffer" during abiotic stress (20). These findings suggest that FHB resistance has a more negative effect on 1000-grain weight and grain weight per spike and a less negative effect on photosynthetic parameters. Therefore, the cost of resistance may be higher in terms of photosynthesis than the cost in terms of yield.

Further analysis of the relationships among $P_{n}, G_{s}$, and $C_{i}$ suggests that the reduced $P_{n}$ in L699, which may be actively regulated (in contrast to passive stomatal regulation), may be an effective physiological mechanism for host FHB resistance. Therefore, the study of the relationship between photosynthetic parameters and yield components may facilitate the elucidation of the mechanism underlying resistance to FHB in wheat. In conclusion, we found that $F$. graminearum infection had greater effects on the photosynthetic parameters of flag leaves and weaker effects on the related yield components, which provides new insight into the consequences of FHB resistance.

\section{Acknowledgments}

We are grateful for financial support that was provided by the National Natural Science Foundation of China (31271721 and 31171557), the Provincial Science and Technology Foundation for International Cooperation Project of Sichuan, China (2010HH0038), the Specific Foundation of Agronomy (No. nyhyzx3-15, 201303016), the Key Project of the Education Ministry of China (2012146), and the Ministry of Science and Technology, China (2011CB100403 and 2013CB127701). We are also grateful for Dr. Hongjie Li of The National Key Facility for Crop Gene Resources and Genetic Improvement (NFCRI), Institute of Crop Science, Chinese Academy of Agricultural Sciences, China, for careful proofread of the manuscript.

\section{Literature Cited}

1. Bai, G., and Shaner, G. 2004. Management and resistance in wheat and barley to Fusarium head blight. Annu. Rev. Phytopathol. 42:135-161.

2. Buerstmayr, H., Steiner, B., Lemmens, M., and Ruckenbauer, P. 2000. Resistance to Fusarium head blight in winter wheat: heritability and trait associations. Crop Sci. 40:1012-1018.

3. Chen, J. B., Liang, Y., Hu, X. Y., Wang, X. X., Zhang, H. Q., Ren, Z. L., and Luo, P. G. 2010. Physiological characterization of 'stay green' wheat cultivars during grain filling stage under field growing conditions. Acta Physiol. Plant. 32:875-882

4. Chen, P., Liu, D., and Sun, W. 1997. New countermeasures of breeding wheat for scab resistance. Pages 59-65 in: Fusarium Head Scab: Global Status and Future Prospects. H. J. Dubin, L. Gilchrist, J. Reeves, and A. McNab, eds. CIMMYT International, Mexico, D.F., Mexico.

5. Del Ponte, E. M., Fernandes, J. M. C., and Bergstrom, G. C. 2007. Influence of growth stage on Fusarium head blight and deoxynivalenol production in wheat. J. Phytopathol. 155:577-581.

6. Ferreira, R. B., Monteiro, S., Freitas, R., Santos, C. N., Chen, Z., Batista, L. M., Duarte, J., Borges, A., and Teixeira, A. R. 2006. Fungal pathogens: the battle for plant infection. Crit. Rev. Plant Sci. 25:505-524.

7. Gilbert, J., and Harber, S. 2013. Overview of some recent research developments in fusarium head blight of wheat. Can. J. Plant Pathol. 35:149-174.

8. Henkes, G. 2008. Plant-microbe interactions alter the allocation of carbon in barley (Hordeum vulgare). Master's Thesis, Fachbereich Biologie, Technische Universitat Darmstadt, Germany.

9. Hill-Ambroz, K., Webb, G. A., Matthews, A. R., Li, W. L., Gill, B. S., and Fellers, J. P. 2006. Expression analysis and physical mapping of a cDNA library of Fusarium head blight infected wheat spikes. Crop Sci. 46:S15-26.

10. Huang, Q., Li, X., Chen, W. Q., Xiang, Z. P., Zhong, S. F., Chang, Z. J., Zhang, M., Zhang, H. Y., Tan, F. Q., Ren, Z. L., and Luo, P. G. 2014. Genetic mapping of a putative Thinopyrum intermedium-derived stripe rust resistance gene on wheat chromosome 1B. Theor. Appl. Genet. 127:843-853.

11. Kong, L., Ohm, H. W., and Anderson, J. M. 2007. Expression analysis of defense-related genes in wheat in response to infection by Fusarium graminearum. Genome 50:1038-1048.

12. Kruger, W. M., Pritsch, C., Chao, S., and Muehlbauer, G. J. 2002. Functional and comparative bioinformatic analysis of expressed genes from wheat spikes infected with Fusarium graminearum. Mol. Plant Microb. Interact. 15:445-455.

13. Liu, Z., Xu, M., Xiang, Z., Li, X., Chen, W., and Luo, P. 2015. Registration of the novel wheat lines L658, L693, L696, and L699, which are resistant to Fusarium head blight, stripe rust, and powdery mildew. J. Plant Regist. 9: 121-124.

14. Lu, W. Z., and Chen, P. D. 2012. Research progress of wheat scab in China Page 5 in: Proceeding of the $4^{\text {th }}$ International Symposium on Fusarium Head Blight, August 23-26. P. D. Chen and H. X. Ma, eds. Nanjing, China.

15. Luo, P. G., Deng, K. J., Hu, X. Y., Li, L. Q., Li, X., Chen, J. B., Zhang, H. Y., Tang, Z. X., Zhang, Y., Sun, Q. X., Tan, F. Q., and Ren, Z. L. 2013 Chloroplast ultrastucture regeneration with protection of photosystem II is responsible for the functional 'stay-green' trait in wheat. Plant Cell Environ. 36:683-696.

16. Luo, P. G., Hu, X. Y., Chang, Z. J., Zhang, M., Zhang, H. Y., and Ren, Z. L. 2009. A new stripe rust resistance gene transferred from Thinopyrum intermedium to hexaploid wheat (Triticum aestivum L.). Phytoprotection 90:57-63.

17. Luo, P. G., Li, X., Xu, M., Yang, J. Z., Zhang, M., Ren, Z. L., and Zhang, H. Y. 2013. A new method for quick identification of wheat Fusarium head blight (Chinese Patent: CN103276047A). http://www.pss-system.gov. $\mathrm{cn} /$ sipopublicsearch/search/search/showViewList.shtml

18. Luo, P. G., Luo, H. Y., Chang, Z. J., Zhang, H. Y., and Ren, Z. L. 2009 Characterization and chromosomal location $P m 40$ in common wheat: a new gene for resistance to powdery mildew derived from Elytrigia intermedium Theor. Appl. Genet. 118:1059-1064.

19. Luo, P. G., Zhang, H. Y., Shu, K., Wu, X. H., Zhang, H. Q., and Ren, Z. L. 2009. The physiological genetic effects of 1BL/1RS translocated chromosome in "stay green" wheat cultivars CN17. Can. J. Plant Sci. 89:1-10.

20. Maydup, M. L., Antonietta, M., Guiamet, J. J., Graciano, C., Lopez, J. R., and Tambussi, E. A. 2010. The contribution of ear photosynthesis to grain filling in bread wheat (Triticum aestivum L.). Field Crops Res. 119:48-58.

21. McMullen, M., Jones, R., and Gallenberg, D. 1997. Scab of wheat and barley: a re-emerging disease of devastating impact. Plant Dis. 81:1340-1348. 
22. Mesterhazy, A. 1995. Types and components of resistance to Fusarium head blight. Plant Breed. 114:377-386.

23. Nganje, W. E., Bangsund, D. A., Leistritz, F. L., Wilson, W. W., and Tiapo, N. M. 2004. Regional economic impacts of Fusarium head blight in wheat and barley. Rev. Agr. Econ. 26:332-347.

24. Nganje, W. E., Johnson, D. D., Wilson, W. W., Leistritz, F. L., Bangsund, D. A., and Tiapo, N. M. 2001. Economic Impacts of Fusarium Head Blight in Wheat and Barley: 1998-2000. Agribusiness \& Applied Economics Reports Department of Agribusiness and Applied Economics, North Dakota State University, Fargo, North Dakota.

25. Prats, E., Carver, T., Gay, A., and Mur, L. 2007. Enemy at the gates interaction specific stomatal responses to pathogenic challenge. Plant Signal. Behav. 2:275-277.

26. Prats, E., Gay, A. P., Roberts, P. C., Thomas, B. J., Sanderson, R., Paveley, N., Lyngkjaer, M. F., Carver, T. L., and Mur, L. A. 2010. Blumeria graminis interactions with barley conditioned by different single $\mathrm{R}$ genes demonstrate a temporal and spatial relationship between stomatal dysfunction and cell death. Phytopathology 100:21-32.

27. Rodriguez-Brljevich, C., Kanobe, C., Shanahan, J. F., and Robertson, A. E. 2010. Seed treatments enhance photosynthesis in maize seedlings by reducing infection with Fusarium spp. and consequent disease development in maize. Eur. J. Plant Pathol. 126:343-347.

28. Rudd, J. C., Horsley, R. D., McKendry, A. L., and Elias, E. M. 2001. Host plant resistance genes for Fusarium had blight: Sources, mechanisms, and utility in conventional breeding systems. Crop Sci. 41:620-627.

29. Shen, X., Liao, H. M., and Luo, L. M. 2005. The reasons for the epidemics of wheat Fusarium head blight in Sichuan in 2004. China Plant Prot. 25:18.

30. Sturm, A., and Tang, G. Q. 1999. The sucrose-cleaving enzymes of plants are crucial for development, growth and carbon partitioning. Trends Plant Sci. 4: 401-407.
31. Swarbrick, P., Schulze-Lefert, P., and Scholes, J. 2006. Metabolic consequences of susceptibility and resistance (race-specific and broad-spectrum) in barley leaves challenged with powdery mildew. Plant Cell Environ. 29: 1061-1076.

32. Wagner, A., Michalek, W., and Jamiolkowska, A. 2006. Chlorophyll fluorescence measurements as indicator of fusariosis severity in tomato plants. Agron. Res. 4:461-464.

33. Withers, C. M., Gay, A. P., and Mur, L. A. J. 2011. Are stomatal responses the key to understanding the cost of fungal disease resistance in plants? J. Sci. Food Agric. 91:1538-1540.

34. Wituszynska, W., Slesak, I., Vanderauwera, S., Szechynska-hebda, M. Kornas, J., Kelen, K. V. D., Muhlenbock, P., Karpinska, B., Mackowski, S. Breusegem, F. V., and Karpinski, S. 2013. Lesion simulating disease1, enhanced disease susceptibility 1 , and phytoalexin deficient 4 conditionally regulate cellular signaling homeostasis, photosynthesis, water use efficiency, and seed yield in Arabidopsis. Plant Physiol. 161:1795-1805.

35. Yao, J. B., and Lu, W. Z. 2000. Progress on breeding for wheat scab resistance in China. J. Jiangsu Agr. Sci. 16:242-248.

36. Zhang, L., Chang, Z. J., Li, X., Zhang, H. Y., Ren, Z. L., and Luo, P. G. 2011 Screen and identification of wheat new resistant germplasms to Fusarium head blight. Acta Phytophylac. Sin. 38:569-570.

37. Zou, J. J., Rodriguez-Zas, S., Aldea, M., Li, M., Zhu, J., Gonzalez, D. O. Vodkin, L. O., DeLucia, E., and Clough, S. J. 2005. Expression profiling soybean response to Pseudomonas syringae reveals new defense-related genes and rapid HR-specific downregulation of photosynthesis. Mol. Plant Microb. Interact. 18:1161-1174.

38. Zwart, R. S., Muylle, H., Bockstaele, E. V., and Ruiz, I. R. 2008. Evaluation of genetic diversity of Fusarium head blight resistance in European winter wheat. Theor. Appl. Genet. 117:813-828. 